\title{
Vrai dialogisme et polyphonie attestée : quand le prêtre parle de son homélie.
}

\author{
Carole Calistri \\ Université de Nice-Sophia Antipolis \\ Laboratoire LIDILEM \\ carole.calistri@unice.fr
}

\section{Pré-ambule}

Activité humaine, activité langagière. Si l'on excepte la partie de l'humanité qui est privée, par naissance ou accident, d'un des sens nécessaires à la communication, on voit le champ immense couvert par les sciences du langage, « langage signe ${ }^{1}$ de l'humain », ainsi que le désigne Malmberg, science de l'humain en communication avec les autres membres de l'espèce, en société. C'est dire également que l'ampleur et la diversité des phénomènes concernés condamnent le linguiste à une certaine " division du travail » linguistique et à ne s'intéresser qu'à un nombre restreint d'objets. Cela ne va pas sans une certaine mutilation : extraire de l'activité humaine l'activité langagière - et ne considérer que parties de celle-ci -, c'est nécessairement se priver de toutes les connections avec de grands pans de réel, que l'on songe ici à la fiction de Miracle en Alabama ou de Johnny s'en va-t-en guerre. Cependant, ainsi que l'écrit Vygostki : « la science [doit] apporte[r][ une]lumière, [ une] clarté sur les questions étudiées en [..] aidant à décomposer, à délimiter les objets, les formes, les phénomènes $[\ldots] »^{2}$

Activité humaine, travail, activité langagière. Ensuite, on pourrait considérer que, activité sociale par excellence, l'activité laborieuse entretient avec l'activité langagière des rapports particuliers: fonctionnements similaires mais aussi interactions. Cela retient aussi l'attention du linguiste de deux manières - au moins ! - différentes :

- $\quad$ qu'en est-il de l'activité langagière produite dans la situation de travail ? par la situation de travail ? quelle articulation?

- qu'en est-il lorsque l'activité langagière est l'activité laborieuse ? lorsqu'il est prescrit de parler?

Quand ce qu'il faut faire, c'est... dire, - si les mânes d'Austin pardonnent le jeu de mots - serait une façon de désigner le domaine de nos intérêts. On comprendra dès lors que le champ professionnel de l'enseignement est concerné au premier chef - mais celui du barreau pourrait l'être tout autant - d'une part, et d'autre part, qu'un certain nombre de métiers convoque l'activité langagière dans le cadre du prescrit, même si cette activité langagière n'est pas l'unique forme d'activité du métier ou même si quantitativement elle n'est pas la plus importante parmi toutes les formes d'activités du métier en question. Un cours, une plaidoirie, une homélie ont en commun cette dimension d'activité langagière prescrite. C'est ce qui intéresse ici. 


\subsection{Premier contexte, contexte ${ }^{3}$ premier : l'auto-confrontation en clinique de l'activité}

\subsubsection{La clinique de l'activité}

On se place ici dans le cadre de l'analyse du travail : la clinique de l'activité est une position que l'on pourrait dire éthique. Le Conservatoire National des Arts et Métiers de Paris est un lieu d'exploration par l'équipe d'Yves Clot, pour le dire un peu rapidement hélas, des rapports de l'homme au travail avec une direction précise: " L'analyse du travail vise toujours, en quelque façon, à comprendre pour transformer $\gg{ }^{4}$ et

[son] objet est moins l'activité comme telle que le développement des activités du sujet et ses empêchements. Dans l'approche historico-psychologique que nous adoptons, le développement d'un sujet n'est d'ailleurs pas une course après un but connu d'avance. Son modèle n'est pas embryologique car le développement n'est unidirectionnel et prédéterminé qu'en dehors des situations réelles. Le réel se charge de transformer le développement attendu en histoire inaccomplie. C'est pourquoi, dans cet ouvrage, [...] nous entendons toujours par développement, l'histoire du développement. Les méthodes permettant d'apprivoiser cet objet relèvent de ce qu'on appellera volontiers une clinique de l'activité

Nous prenons donc le parti, dans notre étude, - cela mériterait un développement autrement argumentatif mais il excèderait la place consentie - de considérer l'homélie comme une activité de travail du prêtre ${ }^{6}$.

Mais il nous faut poser encore un autre cadre conceptuel, pour finir, le genre: appuyé également sur ce que Bakhtine explique du genre :

[...] les formes stables du genre de l'énoncé. Le vouloir-dire du locuteur se réalise avant tout dans le choix d'un genre de discours. Ce choix se détermine en fonction de la spécificité d'une sphère donnée de l'échange verbal, des besoins de la thématique (de l'objet du sens), de l'ensemble constitué par les partenaires, etc. [...] pour parler nous nous servons toujours des genres du discours, autrement dit, tous nos énoncés disposent d'une forme type et relativement stable, de structuration d'un tout. ${ }^{7}$

Yves Clot « [...] appelle genre [...] un corps intermédiaire entre les sujets, un intercalaire social situé entre eux d'une part et entre eux et l'objet du travail d'autre part $»^{8}$.

On voit ici, et cela justifie de poser cette lecture du genre ici, maintenant, se dessiner l'une des pistes d'exploration: en effet, « l'activité langagière est une modalité de l'activité humaine qui peut servir

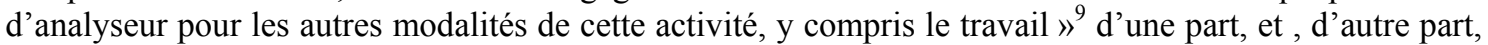
ainsi que « la langue maternelle, [que]nous n'apprenons pas dans les dictionnaires et les grammaires, [que] nous acquérons à travers des énoncés concrets que nous entendons et que nous reproduisons au cours de l'échange verbal vivant qui se fait avec les individus qui nous entourent. Nous assimilons les formes de langue seulement sous les formes que prend un énoncé, et conjointement avec ces formes $»^{10}$, le discours du travail - cette formulation englobe le discours produit en situation de travail et le discours produit sur le travail - est imprégné des mots transmis par d'autres, c'est-à-dire tous les autres: l'allocutaire présent pour qui l'énoncé du locuteur est réponse, mais aussi les autres, ici les pairs dans le travail, qui ont transmis au locuteur des formes et des genres qu'ils avaient eux-mêmes reçus d'autres.

Si nous souhaitons rendre visibles ces emprunts - nous dirons plus loin ce qui motive ce projet -, nous nous heurtons à la difficulté dont Bakhtine lui-même fait état :

Nous possédons un riche répertoire des genres de discours oraux (et écrits). Dans la pratique, nous en usons avec assurance et adresse, mais nous pouvons en ignorer 
difficulté dont on trouve naturellement l'écho en clinique de l'activité, un écho enrichi par la spécificité du domaine, relativement à la position du chercheur d'une part :

l'analyste du travail est précédé "sur ce terrain" par ceux qui y vivent. Il y a rencontre des sujets qui ont déjà dû comprendre et interpréter leur milieu de travail pour lui donner et lui conserver un sens coûte que coûte.[...] expertise brute [...] concepts quotidiens qui organisent l'expérience du travail en question sous des descriptions subjectives formulées dans un vocabulaire souvent métaphorique $»^{11}$

et d'autre part, relativement à la nature des

sédimentation(s) [...] prolongement des activités conjointes antérieures [...] précédent pour l'activité en cours : ce qui a été fait auparavant par les générations d'un milieu donné, les manières dont les choix ont été tranchés jusque-là dans ce milieu, les vérifications auxquelles il a été procédé, les coutumes que cet ensemble rassemble. ${ }^{12}$

On nous pardonnera ces citations pour l'avantage qu'elles procurent de donner sous une forme compacte les éléments nécessaires à l'intelligence de ce qui va être exposé. La question qui est posée maintenant pourrait être la suivante : comment révéler ces traces $?^{13}$ un peu - mais seulement un peu car filer totalement la métaphore conduirait à une vision très différente, presque opposée à celle que nous revendiquons- à la manière dont on révèlerait, grâce à la chaleur de la flamme, des écrits notés avec une encre sympathique. Sur ce point, nous nous appuyons sur les distinctions de Jacques Bres entre phénomènes dialogaux et phénomènes dialogiques - les deux seront, à leur place, examinés ici- et nous présenterons le travail qui suit comme une tentative très modeste de contribution partielle pour dépasser «cet aspect souvent impalpable de la dialogisation [qui] tient non pas au caractère superficiel de l'interaction entre l'énoncé du locuteur et les autres énoncés, mais au contraire à son caractère profond ", phénomènes qui « disposent parfois de marques linguistiques mais c'est loin d'être toujours le cas ${ }^{14}$.

Dans cette perspective - une «co-élaboration chaque fois à refaire d'un instrument d'enquête approprié $»^{15}$, des « formes différenciées de co-analyse du travail ${ }^{16}$ - les méthodes d'autoconfrontation croisée en analyse du travail « fournissent des instruments pour [les] interlocuteurs »: il s'agit de développer des méthodologies d'observation différée :

On peut sédimenter plusieurs traces d'activités « situées », afin que les hommes et les femmes [...] engagés dans l'analyse puissent basculer du statut d' «observés » à celui d'observateurs, co-acteurs dans la production des données recueillies.

C'est ce que la prochaine rubrique présentera ${ }^{17}$.

\subsubsection{L'auto-confrontation}

Ceci aurait également pu s'intituler «mode de recueil des données », car on y trouvera la description de la méthodologie que la partie 2 explorera dans un certain détail. Mais nous commencerons par les principes directeurs :

La méthode en clinique de l'activité propose donc de nouvelles modalités en analyse du travail, où le dialogisme constitue le principe directeur et la source du dispositif méthodologique. Il s'agit, pour l'essentiel, de créer un cadre où les partenaires associés à la recherche, les acteurs concernés par l'activité étudiée, puissent à la fois 


\begin{abstract}
peser sur les attendus et les conditions de cette recherche, et mettre en chantier des manières de penser collectivement leur travail, entre l'image filmée de ce qu'ils ont fait et la mise en discours de ce qu'ils sont en train de faire. Cette démarche institue ce que l'on a appelé un « espace-temps » où les acteurs ont la possibilité de mobiliser, où de rétablir leur "pouvoir d'agir » en différé par rapport aux actions exposées par le film. ${ }^{18}$
\end{abstract}

D'un point de vue pratique, cela s'organise de la manière suivante : il y a trois phases :

- la première consiste en une « conception partagée » : il s'agit de choisir ensemble les situations à retenir pour l'analyse

- la deuxième phase «cumule la production de documents vidéo en auto-confrontation simple sujet/chercheur/images - et de documents d'auto-confrontation croisée - deux sujets/chercheur/ images. C'est le début d'un dialogue professionnel entre deux professionnels confrontés à la même situation ${ }^{19}$

- la troisième phase « [est] un retour devant le « milieu » de travail qui se remet alors au travail d'analyse et de co-analyse ».

Il importe maintenant de fournir un certain nombre de précisions qui seront utiles par la suite :

- « le commentaire des données vidéo de l'opérateur en auto-confrontation sur son travail est adressé, de fait, à un autre que lui $»^{20}$ : ceci renvoie aux phénomènes dialogaux mais " c'est une activité dirigée[...] dans laquelle le langage, loin d'être seulement pour le sujet un moyen d'expliquer ce qu'il a dit ou fait ou ce qu'on voit, devient un moyen d'amener autrui à penser à sentir et à agir selon sa perspective à lui ». Par cette adresse à la personne du chercheur et/ou du pair, le commentaire de l'opérateur sera modifié mais
loin de traiter ces phénomènes comme un obstacle, nous proposons d'en faire un atout méthodologique [...] le sujet ne cherche pas d'abord en lui-même mais dans l'autre. D'une façon ou d'une autre, il lutte contre une compréhension incomplète de son activité par ses interlocuteurs, il soupçonne en eux cette incompréhension, cette insuffisance, et veut la prévenir. Il vise à s'approprier pour les modifier leurs mobilisations respectives à propos de son travail et, du coup, il voit sa propre activité « avec les yeux » d'une autre activité. Il éprouve, déchiffre et parfois développe ses émotions par l'entremise des émotions d'autrui. ${ }^{21}$

Et quand cet autrui change - le chercheur, le pair, plus tard le groupe des pairs, le collectif -, l'opérateur regarde son activité par le prisme de deux autres activités qui peuvent être discordantes. L'autoconfrontation croisée utilise ces dissonances. Peut-être peut-on voir ici l'une des raisons qui donnerait des moyens de s'approcher de la profondeur de l'interaction entre l'énoncé du locuteur et les autres énoncés.

- Le second élément regarde ce que Daniel Faïta et Yves Clot appellent la motricité du dialogue (on donne ici la «correction » de la métaphore concernant l'encre sympathique) : le ressort dialogique qui soutient l'auto-confrontation croisée est précisément... dynamique, c'est-à-dire vivant, c'est-à-dire imprévisible et mouvant.

[l'autoconfrontation] organise le refus de tout discours achevé. C'est pourquoi on pourrait penser, à la manière de Bakhtine, que le genre dialogique de l'autoconfrontation croisée vise surtout à contaminer l'activité ordinaire, non pour la soumettre à des canons qui ne sont pas les siens mais pour l'affranchir de tout ce qui est conventionnel, nécrosé, ampoulé, amorphe, de tout ce qui freine sa propre évolution. Car « il reste toujours un excédent d'humanité non réalisé » écrit Bakhtine. $^{22}$ 
Il s'agit de dire que ce que l'auto-confrontation « révèlera » n'est pas de l'ordre d'un inscrit préalable et fixé, antérieur ou caché, mais que, pour partie, ceci se construit dans/ par l'interaction dialogique. Le projet pour cette communication vise un but qui est, de beaucoup, moins ambitieux que celui poursuivi par les auto-confrontations qui visent à développer le pouvoir d'agir d'interlocuteurs qui ont fait appel à l'équipe de la clinique de l'activité pour modifier, à leur demande, des situations réelles de travail dégradées ; il se bornera à un aspect de la première phase - qui analyse l'activité concrète -, dans laquelle se trouve l'activité langagière, transformer pour comprendre.

On pourrait encore convoquer ici tout le chapitre intitulé " facile à dire, difficile à dire » de Frédéric François dans Le discours et ses entours, "La diversité des mises en mots a une certaine relation (prudence) à la diversité des façons réelles d'être ou de sentir. Même si tantôt on saisit le langage comme possibilité de rendre présent ce qui ne peut être directement objet d'expérience ou au contraire la diversité du réel expérimenté comme ne pouvant jamais être dite. ». A tout le moins, on ne confondra pas le réalisé avec le réel. C'est le dernier point que traitera la partie suivante.

\subsubsection{Le réel de l'activité : possibles et dialogisme}

Yves Clot le définit en ces termes :

Le réel de l'activité, c'est aussi ce qui ne se fait pas, ce qu'on ne peut pas faire, ce qu'on cherche à faire sans y parvenir - les échecs - ce qu'on aurait voulu ou pu faire, ce qu'on pense ou qu'on rêve de pouvoir faire ailleurs. Il faut y ajouter - paradoxe fréquent - ce qu'on fait pour ne pas faire ce qui est à faire ou qu'on fait sans vouloir le faire. Sans compter ce qui est à refaire. ${ }^{23}$

Si on peut ainsi caractériser le réel de l'activité concrète, il pourra donc en être de même pour l'activité langagière qui est une partie de cette activité concrète, et permettre de distinguer entre dialogue réalisé et réel du dialogue, " interférence consonante ou dissonante des répliques du dialogue apparent avec les répliques du dialogue intérieur $»^{24}$. En reprenant, d'une autre manière ce qui a été évoqué plus haut, c'est cet hétérovocalisme, ce « dur combat dialogique » que l'on se propose de tenter de saisir grâce à l'autoconfrontation réalisée.

\section{Second contexte : une homélie dominicale}

Il a été choisi d'utiliser la méthodologie de la clinique de l'activité sur une homélie dominicale ${ }^{25}$ : les deux prêtres de la paroisse ont prêché, l'un à la messe anticipée du samedi soir, l'autre à la messe du dimanche, sur les mêmes textes, pour une fête liturgique d'une importance relative - Trinité - mais sans l'éclat de la Nativité ou de Pâques, il ne s'agissait donc pas d'une prédication extra-ordinaire.

\subsection{Intérêt de l'objet homilétique}

D'une certaine façon, le défi était de saisir le vivant, le mouvant, l'imprévisible dans un genre assez fixé de beaucoup de manières différentes : on pourrait penser à la première de ces mises en forme constituée par la préparation car, pour les homélies dominicales, si peu de prêtres lisent une homélie entièrement rédigée, presque tous en revanche viennent à l'ambon avec au moins quelques notes, on peut penser aussi à la fixation-fixité chronologique établie par la liturgie, l'homélie intervient après la proclamation de l'Evangile et des lectures du jour, elle ne peut être déplacée, elle peut être en revanche omise dans les messes de semaine - à l'intérieur d'un cadre ancien d'au moins quinze siècles- la messe - où ce qui porte le nom de dialogue ressemble assez peu à une conversation. Il y a également les contraintes qui pèsent sur sa durée - l'homélie trop longue allonge la messe, dont les parties qui suivent ne peuvent être écourtées -, sur son ton communicationnel - la familiarité comme la trop grande distance ne peuvent être choisies sans risque- sur son contenu - prioritairement l'Evangile, puis les lectures, les fêtes et l'actualité religieuse locale ou internationale -. Bref, un genre à la forme, si l'on reprend à Bakhtine son expression, plus que stable... 


\subsection{Dialogue et dialogisme dans l'homélie, polyphonies ?}

On s'est conformé à la méthodologie de l'auto-confrontation simple tout d'abord - chacun des prêtres a visionné et commenté avec le chercheur le film de son homélie, ensuite les deux prêtres le chercheur ont vu chacun l'homélie de l'autre : on a donc trois textes - au sens que donne Bronckart « toute production verbale située, qu'elle soit orale ou écrite ${ }^{26}$ recueillis :

- celui de l'homélie : texte 1

- celui de l'auto-confrontation simple : texte 2

- celui de l'auto-confrontation croisée : texte 3

\subsubsection{La question de l'unité d'analyse}

L'unité est celle de l'interaction, au sens donné par Robert Vion, la question est toujours posée de la légitimité de «prélèvement» dans un ensemble «tissé » par l'articulation des tours de parole. C'est une question qui a un versant technique - ici par exemple la faisabilité/accessibilité à une intelligence correcte des textes sans en avoir la totalité disponible - sans pour autant perdre la dimension théorique : comment décrire/analyser les textes 2 où, par la présence de la vidéo, se trouve convoquée l'assemblée paroissiale mais où également le prêtre entre en dialogue avec un lui-même, qui n'est plus tout à fait celui qui regarde la vidéo.

Car en effet, ce qui advient au tour de parole par exemple 77, n'advient que parce que 76 autres tours de parole l'ont précédé, comme préparé. Cependant, en regardant les traces que nous recherchons, et la particularité de la mise en œuvre, on pourra convenir - parce qu'il faut prendre un parti - que la paire, « adjacente» en quelque sorte, formée par l'élément considéré comme saillant par l'opérateur dans son premier discours, au point de susciter une réaction et cette réaction elle-même, a une cohérence suffisante pour être une unité convenable à analyser (par ailleurs la numérotation des tours de parole donne une indication sur l'emplacement chronologique et on mentionnera, si le besoin se manifeste, les éléments antérieurs, non compris dans la paire, nécessaires à l'interprétation ${ }^{27}$ qui pourraient utilement éclairer)

\subsubsection{Quelques exemples}

Petit préambule de présentation : pour la commodité du repérage des différents textes, le texte $\mathbf{1}$ est en italique souligné, ses lignes sont numérotées afin que l'on puisse avoir une indication sur le moment (l'homélie a duré 11 minutes et 45 secondes, 91 lignes) choisi.

\section{- Dialogisme interdiscursif et intralocutif}

1. La Très Sainte Trinité +++ la Trinité + ce n'est pas ++ une invention humaine ++ un concept de 2.théologien++ fêter la Trinité++ c'est vraiment aller au cour /de la foi chrétiennel et je dirais même/ de 3.la spécificité de la foi chrétienne++ souvent quand on parle de Trinitél on voit quelque chose de 4.compliqué/ quelque chose qu'il faut réserver pour les gens intelligents ++

Lors de l'auto-confrontation simple, spontanément le prêtre demande d'interrompre le film à cet endroit et dit

33.PJG ${ }^{28}$ : alors voilà le truc/ je parlais de gens/ à réserver aux gens intelligents/ je suis pas d'accord finalement sur ça parce tu vois/ je dis souvent dans mes prédications et là je le dis mais / c'est pas exactement ça que je voulais dire/ c'est euh être chrétien demande qu'on soit intelligent/ donc c'est un peu le contraire que j'ai dit/ en disant ben voilà c'est pas réservé aux gens intelligents/ vous n'êtes pas intelligents et--/ au contraire/ le mot c'était pas c'était pas le mot intelligent sur lequel/ XXX c'est pas c'est pas une spéculation--- de l'esprit et cætera/ par contre l'intelligence on en a besoin/ ça fait partie de du pacte du chrétien pour-- pour percer le mystère de Dieu/ il faut bien l'exercer/ la foi/ requiert aussi l'intelligence/ c'est intelligent d'avoir la foi 
Soi-même comme un autre ... L'opérateur dit: je ne suis pas d'accord avec moi-même, ou plutôt je n'aurais pas été d'accord avec ce que j'entends ici si j'avais été un auditeur dans l'assemblée. Le raisonnement lors de l'homélie constituerait assez facilement un Face Threatening Act : "l'explication », pour le dire vite, de la Trinité ne s'adresse pas, contre les apparences, uniquement aux gens intelligents, donc même si vous êtes bêtes, c'est pour vous, FTA augmenté par l'obligation qu'a le prêtre de prêcher sur cette question (c'est la suite immédiate : mais si je ne parle pas aujourd'hui de la Trinitél je ne parle pas non plus du Dieu des chrétiens +++ et j'y suis obligé par ma charge !).

Or ce qui est « plié » pourrait-on dire dans l'homélie, se déplie, déploie dans l'auto-confrontation simple où l'opérateur retrouve, ré-organise à la fois son intention du moment de l'homélie - il était bien question comme on en trouve un certain nombre de traces dans la suite de rassurer les auditeurs qu'il a imaginé effrayés par le caractère ardu " intelligent ", du sujet: Dieu Trine, en trois personnes distinctes mais formant une Unité - et une autre possibilité de raisonnement qui s'ouvre à lui avec cet émergé : pour tout ce qui regarde la foi, l'intelligence peut et doit être sollicitée, que vous soyez intelligents ou non, rompus aux finesses théologiques ou non, la foi - et vous l'avez! vous commande en quelque sorte de chercher des ressources dans votre intelligence.

Ce qui, on doit l'avouer, permet, - d'une manière plus satisfaisante, au moins pour la préservation de la face, que ce qui est fait dans l'homélie - de prendre en compte le réseau des contraintes :

- il faut prêcher sur la Trinité,

- l'explication, si elle est nécessaire liturgiquement - il faut tenir compte de cette fête - n'en est pas moins facile intellectuellement,

- tous les paroissiens ne sont pas des théologiens, même débutants, or suivant un Père de l'Eglise, «Les prêtres dans l'Église ressemblent aux vaches et le peuple chrétien représente en figure les veaux. De même que les vaches courent de tous côtés par les champs et les prés, font le tour des vignobles et des olivettes pour brouter herbes et feuillages et en préparer le lait qui nourrira leurs veaux, de même les prêtres, en lisant assidûment la Parole de Dieu, doivent cueillir les fleurs sur les divers monts des Écritures Saintes, pour pouvoir en extraire un lait spirituel et le servir à leurs fils ${ }^{29}$

Avec cet exemple, nous rejoignons la réflexion d'Yves Clot sur «ce "difficile à dire" avec quoi on pourrait peut-être faire quelque chose de différent de ce qu'on fait». Il nous semble saisir ici quelque chose qui permet de dépasser " l'aller-retour sans surprise entre un réel dialogique saturé de voix mais inaudible et un dialogue réalisé bruyant mais monocorde $»^{30}$, car en rendant audibles, par le truchement de l'auto-confrontation, des voix peut-être tues, on élargit les possibles, le pouvoir d'agir.

Un autre exemple va montrer un autre fonctionnement :

44. Lui/ ce Dieu Trinité et qui va créer/non pas l'homme comme ça geste spiralaire vers le haut/ comme 45. un absolu/mais qui va créer en même temps le couple humain+ comme image geste de projection de 46. Dieu++c'est merveilleux de découvrir cela +++ en Dieu- le Père aime le

Commenté ensuite de la façon suivante

134. PJG : je suis parti là-dessus

135. Ch : et à votre avis pourquoi vous êtes parti là-dessus ?

136. PJG : c'est à force de préparer les gens au mariage rires tout simplement

137. Ch : voilà

Il ne s'agit pas ici d'un difficile à dire mais d'un effet de «traçabilité » pourrait-on dire et curieusement ceci est anticipé dans le commentaire qui termine la précédente séquence

133. PJG : je pense que je me suis laissé prendre par la phrase que j'ai dite ensuite que je comptais pas forcément dire parce que parler de l'homme et de la femme c'était pas obligé/ j'avais ça en tête bien sûr parce que la/ le couple humain est créé à l'image de Dieu donc est fait pour l'amour et va donner l'amour/ mais il y avait pas lieu cette fois/ j'aurais dû laisser ça de côté/ et j'aurais XXX l'autre idée 
Le sentiment d'incongruité relative et la perplexité de constater un développement non absolument exigé sont éclaircis, grâce à l'auto-confrontation, par le fait de retrouver, dans ce discours " bizarre » de quoi l'expliquer néanmoins : c'est à force de préparer les gens au mariage. De façon très intéressante, ce point sera aussi relevé dans l'auto-confrontation croisée par le pair. Les échanges et l'articulation entre le premier discours - l'homélie- et son commentaire - sont moins resserrés du fait de la modification du cadre de l'interaction (trois personnes au lieu de deux), donc l'extrait qui suit ne se trouve pas immédiatement après le moment où le film a été arrêté.

197. PPJ : [à PJG] non moi ce que je pense c'est que tu es quand même tu es quand même plus à l'aise sur l'argumentation--- euh l'homme à l'image de Dieu dans le couple/ tu as toute la prépa mariage dessus et tu glisses d'une manière un peu facile rapidement dessus là où tu es plus à l'aise quoi et tu as peut-être même pas de quoi argumenter plus sur le Faisons l'homme à notre image

198. PJG : oui mais si j'avais en préparant pris le temps de ça d'en parler tu vois

199. Ch : hé bien faites-le maintenant/ si vous aviez pu aller jusqu'au bout de l'argumentation/ c'était Faisons Il n'est plus tout seul/ curieusement d'ailleurs vous dites Il revient sur Lui-même/ ce qui est

200. PJG : oui je trouve l'expression un peu maladroite

201. Ch : qu'est-ce que vous vouliez dire?

202. PJG : non c'est c'est--- quand on est plusieurs on fait un colloque on dit allez geste du bras arrondissant-rassemblant maintenant on se met ensemble et on va faire tu vois

203. Ch : hmhm

204. PJG : et dans ce sens-là y a

205. Ch : une réunion de plusieurs

206. PJG : réunion/ mais c'est pas un retour sur soi/ ça donne une idée fausse effectivement/ en l'entendant je dis c'est pareil/ mais là il y a le changement de ton dans le verbe tu vois dans ce pluriel qui est lu/ dans la Tradition chrétienne comme l'action de toute la Trinité au moment où justement on crée l'homme à l'image de Dieu et cette image d'un Dieu trinitaire justement puisqu'en face c'est le pluriel c'est l'homme pluriel

L'intervention du pair - qui, remarquons-le au passage ne ménage pas la face de son curé et tu as peutêtre même pas de quoi argumenter plus sur le Faisons l'homme à notre image - et celle du chercheur engagent l'opérateur sur une réalisation qui actualise une activité empêchée au moment de l'homélie.

\section{- Dialogisme interlocutif}

18. baptisez-les/ au nom du Père+ du Fils + et du Saint-Esprit +++ nous devons tous avoir cette conviction 19.quel la Trinité n'est pas/ une élucubration fumeuse de théologiens/mais c'est bien comme cela/ que 20.Dieu nous a parlél si nous sommes chrétiens + nous croyons/à la Très Saintel Trinité+ à notre Dieu qui 21.est à la fois Père/Fils/ et Saint-Esprit++ un seul Dieu++en

Ce passage est commenté de la façon suivante dans l'auto-confrontation simple

46. PJG : mais alors c'est toujours pareil pour le---par exemple le débit/ est-ce qu'on comprend bien/est-ce que--c'est pas trop rapide les mots/ même chose les gestes à chaque-frappe la table avec le poignet/ est-ce que c'est pas trop

D'une manière plus attendue et plus classique, on entend ici une autre voix que celle qui s'exprime dans l'homélie, celle qui inscrit le souci de l'autre comme auditeur, sur des points relatifs à la production physique-physiologique du discours, mais ailleurs cela peut être, et comme l'indiquait le premier exemple, sur des questions de compréhension.

Il y a un autre exemple de dialogisme interlocutif qui mérite qu'on s'y arrête pour la preuve qu'il donne que, comme l'écrit Vygostki, l'homme est plein à chaque instant de possibles non réalisés. 
50.fruit de cet amour++ mieux encore/ l'homme/ est créé geste ponctuant à l'image de ce Dieu Amour/ et 51.qui ne peut pas faire autrement que de se communiquer/ que de se donner++ nous avons ici--le secret 52.de toute existence humaine ++ l'homme est créé par amour et pour l'amour/ et il trouve dans la Trinité 53.elle-même le modèle de ce qu'il est/ la source de ce qu'il est+++ l'homme est créé pour réaliser--ce 54.projet d'amour sur lui/ cette communication inter-personnelle à l'image de--celle que le Père a pour le 55.Fils et que le Fils a pour le Père +++ et la famille humaine doigts rejoints vers le haut est précisément $+56 . c e l a+++$ quand on parle de--la famille certains spirituels ont dit ben c'est une petite Trinité sur la 57.terre++ les époux qui s'aiment/ et cet amour qui est lui-même fécond ++ c'est pas faux/ c'est même 58.tout à fait juste

Lors de l'auto-confrontation simple, l'opérateur indique

160. PJG : je sais plus pourquoi je parlais de ça/ c'était la fête des mères aussi/ hein c'est ça?

161. Ch: XXX

162. PJG : c'était la fête des mères

163. Ch: le 7 ?

164. PJG : si si c'était la la fête des mères sinon j'aurais pas parlé de ça

Et c'était en effet la fête des mères - comme cela sera écouté ensuite - mais lors de l'auto-confrontation croisée

217. PJG : y a aussi /c'est l'actualité qui m'a amené à parler de ça je pense/ on devait être dans les lois sur la bio-éthique

218. Ch : hmhm

219. PJG : et donc c'est pour ça qu'il y a ce petit chapitre sur la famille qui venait bien à ce moment-là/ les familles trinitaires

220. Ch : ben c'est sûr que

221. PJG : du coup j'avais tendance en le faisant je me suis dit holà tu t'éloignes un petit peu/ il faudrait bien bien tenir le tout

Bien sûr, on peut toujours tenir l'argument d'une mémoire défaillante - cependant, immédiatement avant le début de l'auto-confrontation croisée, l'homélie a été re-visionnée entièrement - mais ce qui est plus intéressant est de voir cette variété particulière de prolepse pourrait-on dire, surgie à la faveur de l'autoconfrontation croisée, absente dans l'auto-confrontation simple, mais reliée très nettement à une mention sans ambiguïté à cet égard plus loin dans l'homélie

72. l'image de ce Dieu Trinité/ nous percevons aujourd'hui combien/la famille humaine et la femme qui 73. tient au milieu de cette famille un rôle tout à fait particulier/doit être--je vais dire pas seulement 74.défendue mais + promue/ mise comme-- une lampe geste "éclairant 》 avec la main droite prophétique 75.pour notre temps d'aujourd'hui où cette réalité est si souvent niée

On pourrait encore repérer un grand nombre de ces inscriptions de l'autre dans les discours tenus, comme les mentions des auteurs des études au séminaire, ou telle homélie entendue dans l'enfance, mais il fallait faire un choix et on a choisi de retenir les éléments les plus saillants pour notre projet ${ }^{31}$.

\section{En guise de conclusion}

Tout se passe comme si ces dialogues, dans leur mise en œuvre à l'artificialité revendiquée, permettaient de tirer de certains fils rouges souterrains, issus des « couches profondes ». Au-delà de la satisfaction qu'apporte d'une part une forme d'appropriation - Frédéric François écrit « il fait partie de la façon d'être des hommes de devoir refaire par eux-mêmes un mouvement déjà fait par d'autres. On peut se dire que chacun reprend, accentue à sa façon une expérience commune » - et, d'autre part, du plaisir intellectuel de prendre part à l'un d'un des nombreux (petits) défis laissés aux détours de leur œuvre par des auteurs à la pensée puissamment heuristique, ainsi que l'est Bakhtine, il reste que cette recherche des 
marques dialogiques ouvre sur les champs et les chantiers plus vastes du développement humain articulé sur une solidarité de fait ${ }^{32}$ :

C'est que, comme l'écrit Ponzio (1998), le dialogue n'est pas le résultat d'un acte délibéré et intentionnel d'ouverture à l'autre mais résulte tout au contraire de l'impossibilité de se fermer à l'altérité, sinon à s'exposer aux risques de la maladie mentale. On ne choisit pas de s'ouvrir à l'altérité dialogique. Elle nous affecte avant même de vivre et d'agir dans le monde. Elle est insupprimable. On peut, au mieux, retournant la passivité en activité, en faire quelque chose pour soi en la développant. Sinon c'est elle qui nous enveloppe, non sans nous faire courir le risque d'une déréalisation. On n'y parvient qu'en réussissant à renverser le statut de l'autre, par une subversion de l'autre en soi-même pour soi-même, par appropriation ${ }^{33}$.

\section{Références bibliographiques}

Bakhtine, M. (1984). Esthétique de la création verbale. Paris : Gallimard.

Bres, J. Haillet, P.P., Mellet, S., Nolke, H., Rosier, R. dir. (2005). Dialogisme et polyphonie, approches linguistiques. Actes du colloque de Cerisy, Bruxelles : De Boeck-Duculot.

Bronckart, J.-P. (1996). Activité langagière, texte et discours, pour un interactionnisme socio-discursif. Lausanne : Delachaux et Niestlé.

Clot, Y. (1999). La fonction psychologique du travail. Paris : PUF.

Clot, Y. (2008). Travail et pouvoir d'agir. Paris : PUF.

Faïta, D. (1989). Mondes du travail et pratiques langagières. Langages, $n^{\circ} 93,110-123$.

Faïta, D. et Clot, Y. (2000). Genres et styles en analyse du travail. Concepts et méthodes. Travailler, $n^{\circ} 4,7-42$.

François, F. (1998). Le discours et ses entours. Paris : L’Harmattan.

Goffman, E. (1973). La mise en scène de la vie quotidienne. Paris : Minuit.

Goffman, E. (1974) Les rites d'interaction. Paris : Minuit.

Goffman, E. (1987). Façons de parler. Paris : Minuit.

Kerbrat-Orecchioni, C. (1998). Les interactions verbales. Paris : Armand Colin.

Kerbrat-Orecchioni, C., (2005). Le discours en interaction. Paris : Armand Colin.

Ponzio, A. (1998). Alterita, responsabilita e dialogo in Michail Bachtin. In Bachtin, Per una filosofia dell'azione responsible, Lecce : Piero Manni.

Ponzio, A. (2009). L'écoute de l'autre. Paris : L'Harmattan.

Ponzio, A. (2010). Rencontres de paroles, L'autre dans le discours. Paris : Alain Baudry et Cie.

Vion, R. (1992-2000). La communication verbale, analyse des interactions. Paris : Hachette.

1 C'est nous qui soulignons. Le langage signe de l'humain, Bertil Malmberg, Collection Empreinte, Picard, Paris, 1979.

${ }^{2}$ Vygostki, Lev. Conscience inconscient émotions, Vygostki, la conscience comme liaison, par Yves Clot, Paris : La Dispute, 2003, p.68

${ }^{3}$ Peut-être est-il bon de préciser que contexte est pris ici dans son sens commun, non référé à telle ou telle école linguistique.

${ }^{4}$ Clot, Y. La fonction psychologique du travail, Paris : PUF, collection Le travail humain, p. 2 
${ }^{5}$ idem, p. 3

${ }^{6}$ Un travail pionnier en la matière peut autoriser cette catégorisation-requalification, il s'agit du mémoire de Laure Kloetzer, Analyse de l'Homélie de la messe dominicale : langage et conflits du métier dans l'activité des prêtres. La part de Dieu, la part de l'Homme, soutenue en avril 2008 au CNAM, dans la spécialité Psychologie du travail.

${ }^{7}$ Mikhaïl Bakhtine, Esthétique de la création verbale, Paris : Gallimard, p. 284

${ }^{8}$ Clot, Y. op.cit. p. 34

${ }^{9}$ Clot, Y. idem. p.36

${ }^{10}$ Mikhaïl Bakhtine, Esthétique de la création verbale, Paris : Gallimard, p. 285

${ }^{11}$ Clot, Y.op.cit. p. 134

${ }^{12}$ Clot, Y. idem. p. 37

${ }^{13}$ La perspective nous semble différente de celle qui organise les élicitations, telles que A. Cicourel les expose dans "Elicitation as a Problem of Discourse» (Sociolinguistics: an international handbook of the science of language and society, Berlin et New-York: Walter de Gruyter, 1988, p.1013-1022). En effet, certes le " contrôle de l'échange », qui appartient à l'interviewer d'un homme politique en vue, au policier, à l'avocat, au juge qui interroge témoins, suspects ou criminels ou au médecin en instance de diagnostic qui questionne un patient, appartient également également au chercheur dans l'auto-confrontation [Robert Vion dans la typologie qu'il propose pour les interactions distingue lui entre l'entretien (interview/enquête dans lequel le questionneur est seul au fait des enjeux et capable du décryptage des réponses), la consultation (dans laquelle l'expert rend au consultant le service pour lequel il vient : le conseil) et la transaction (vendeur/client)] mais il faut souligner que, dans le protocole classique, l'acteur a été associé au processus de sélection de l'activité qui sera filmée, qu'il ne répond pas uniquement sur sollicitation du chercheur mais qu'il est libre de le faire spontanément, autant de fois et aussi longtemps qu'il le désire, qu'ensuite dans l'auto-confrontation croisée, c'est l'échange avec le pair qui intéressera tout autant et que le «contrat » avec le chercheur est aussi transparent que possible : en principe son intervention a été souhaitée et il a indiqué l'option de la controverse - pour élargir les possibles - Enfin, le choix d'un appui vygostkien qui tient la conscience pour une liaison ( « expérience vécue d'une expérience vécue » en particulier grâce au langage) s'accommode difficilement de l'idée d'un savoir tapi et rapté à l'insu de l'acteur.

14 Jacques Bres, "Savoir de quoi on parle: dialogue, dialogal, dialogique, polyphonie... », dans Dialogisme et polyphonie, approches linguistiques, Bruxelles : De Boeck-Duculot, Actes du colloque de Cerisy, 2005. p.53 et 55.

${ }^{15}$ Clot, Y.op.cit. FPT, p.136

${ }^{16}$ Clot, Y. idem, p.133

17 De ce point de vue, l'instrument méthodologique de la clinique de l'activité n'est pas comparable à ceux mis en oeuvre dans les travaux de Gumperz (1982/1989 pour la traduction française) concernant les indices de contextualisation - ou même la correction qu'Auer imprime à la définition de Gumperz dans sa fameuse introduction à The contextualization of language (1992) - dans la mesure où ni Gumperz ni Auer ne revendiquent une coconstruction du savoir ainsi bâti et que ce dernier n'a pas pour finalité de nourrir les ressources de la professionnalité des « interviewés/enregistrés ».

18 «Réflexions méthodologiques sur l'auto-confrontation croisée », Daniel Faïta et Marcos Vieira, revue Skhole, hors série 1, 2003.

19 " Genres et styles en analyse du travail. Concepts et méthodes », Yves Clot et Daniel Faïta, revue Travailler, 2000 - 4, p.26

${ }^{20}$ Clot, Y. FPT p. 143

21 idem, p. 143

${ }^{22}$ Clot, Y. (2008), Travail et pouvoir d'agir, Paris : PUF, « Provoquer le dialogue : l'auto-confrontation croisée », p. 230 .

${ }^{23}$ La fonction psychologique du travail, Collection Le travail humain, PUF, 1999-2004, p. 119 
${ }^{24}$ Clot, Y. (2008), Travail et pouvoir d'agir, Paris : PUF, « Provoquer le dialogue : l'auto-confrontation croisée », p. 205.

${ }^{25}$ Voir note 30 .

26 Bronckart, J.-P. (1996), Activité langagière, texte et discours, pour un interactionnisme socio-discursif, Lausanne : Delachaux et Niestlé, p.73.

${ }^{27}$ Nous employons ce terme en référence à la justification qu'en donne Frédéric François dans Le discours et ses entours.

${ }^{28}$ L'auto-confrontation simple a duré 39 minutes et 28 secondes et il y a eu 192 tours de parole (interventions suivant la définition donnée par Robert Vion). Le tour 33 est à 4 minutes et 8 secondes du début. PJG désigne le premier prêtre, PPJ le second et $\mathrm{Ch}$. le chercheur. Le codage est assez transparent mais on le donne ici par sécurité : + désigne les pauses, le nombre de + correspond à la longueur des pauses, la barre oblique désigne des micro-ruptures, les chevauchements de paroles sont soulignés, les petits traits accrochés aux finales le fait que le locuteur prolonge le son, XXX désigne des mots inaudibles, les indications autres sont données en italiques à l'intérieur de l'énoncé.

${ }^{29}$ Césaire d'Arles (470-542), Sermons au peuple 4, 4, SC 175, Cerf, Paris 1971, p. 299-301

${ }^{30}$ Clot, Y. (2008), Travail et pouvoir d'agir, Paris : PUF, « Provoquer le dialogue : l'auto-confrontation croisée », p. 219

${ }^{31}$ Peut-être pourra-t-il être dit ici qu'il n'est pas tout à fait indifférent à un linguiste, qu'il soit intéressé ou non personnellement ou intellectuellement, aux choses de la foi, de travailler - même à distance homilétique - sur des textes sacrés qui recèlent ces deux phrases : "Au commencement était le Verbe » $(\mathrm{Jn}, 1,1)$ et « Dieu dit [...] et il en fut ainsi» $(\mathrm{Gn}, 1,14)$, non pas acte de parole mais acte-parole...

${ }^{32}$ L'espace bref d'une communication ne permet pas de considérer longuement des champs autres que celui des linguistiques qui pourraient accueillir les prolongements d'un tel type de travail, mais il est assez naturel d'envisager que les sciences religieuses y aient leur place (la fonction « talmudique » en quelque sorte de l'homélie, une certaine compréhension de l'exégèse patristique, certains éléments de théologie dogmatique et surtout la « pastoralité » de la doctrine).

${ }^{33}$ Clot, Y. (2008), Travail et pouvoir d'agir, Paris : PUF, « Provoquer le dialogue : l'auto-confrontation croisée », p. 209 